\title{
EVIDENCE FOR A SUPERMASSIVE OBJECT IN THE NUCLEUS OF THE GALAXY M87 FROM SIT AND CCD AREA PHOTOMETRY
}

\author{
Hale Observatories, California Institute of Technology, Carnegie Institution of Washington \\ AND \\ FREDERICK P. LANDAUER \\ Space Photography Section, Jet Propulsion Laboratory \\ Received 1977 June 10; accepted 1977 October 7
}

Peter J Young, James A. Westrhal, Jerome Kristian, and Christopher P. Wilson

\begin{abstract}
Two-dimensional SIT and CCD detectors have been used to measure the surface brightness of the peculiar elliptical radio galaxy M87. Measurements were made in three broad-band colors $(B, V$, and $R)$ to a distance of $80^{\prime \prime}$ from the nucleus, with $1^{\prime \prime}$ spatial resolution and photometric accuracy of the order of $1 \%$.

The data are given in some detail and are compared with earlier photographic results. The most obvious feature of the data is a bright, barely resolved central luminosity spike, which is not seen in similar data on other nearby normal ellipticals. Also, attempts to fit isothermal or King models away from the nuclear spike show additional excess luminosity in the central regions of the galaxy $\left(r<10^{\prime \prime}\right)$, which cannot be fitted by such a model.

A model-independent dynamical analysis, using the photometric data combined with spectrographic results by Sargent et al., shows that the nucleus of M87 contains a compact mass of low luminosity, with $M=5 \times 10^{9} M_{\odot}, r<100 \mathrm{pc}$, and $M / \mathscr{L}>60$. All of the existing data is well fitted by a King model containing a central black hole of mass $M=3 \times 10^{9} M_{\odot}$ and a point luminosity source. While such a model is not uniquely required by the data, it is perhaps the most plausible of several possible models considered. At present, M87 is probably the best case for a hypothetical massive black hole in a galaxy nucleus.
\end{abstract}

Subject headings: galaxies: individual — galaxies: nuclei — galaxies: photometry galaxies: structure

\section{INTRODUCTION}

Theoretical attempts to understand quasars, radio galaxies, and active galaxy nuclei have demonstrated the attractiveness of a gravitational source of energy, such as a black hole (e.g., Lynden-Bell 1969). The mass of the central object is thought to be at least $10^{8} M_{\odot}$, which is the mass equivalent of the total energy output of a quasar or radio galaxy, and might be much higher if the processes that convert mass to energy are inefficient. An object with this mass would have observable effects on the dynamics of the galactic nucleus in which it is embedded. These effects may be probed with optical observations of luminosity profiles and absorption-line widths to detect a bright central "cusp" of stars bound to the massive object and to observe an increased stellar velocity dispersion within this cusp.

The peculiar E0 galaxy M87 (=NGC $4486=$ Arp $152=3 \mathrm{C} 274=$ Virgo A) appears to be a particularly promising candidate for a black hole or supermassive object quest. It is relatively close, it is known to be an active (core-halo) radio source with compact central components (Cohen et al. 1969; Kellermann et al. 1973), and there is evidence for ejection of mass from the nucleus in the form of the optical jets (Felten 1968; Felten, Arp, and Lynds 1970). The total anomalous radiation output from the galaxy is $\sim 2 \times 10^{42} \mathrm{ergs} \mathrm{s}^{-1}$ (Moffet 1975), which places it among the weaker of the supergiant elliptical galaxy radio sources.

In this paper and another (Sargent et al. 1978), photometric and spectroscopic observations of M87 are presented that have been successful in detecting a supermassive object in the nucleus of this galaxy. The mass of the object is $\sim 5 \times 10^{9} M_{\odot}$, which causes large and easily observable effects on the dynamics of the nucleus of M87.

Here we present the photometric data obtained with digital SIT and CCD systems on the Palomar 60 inch and 200 inch telescopes. In $\S$ II we discuss the observations and the reduction procedures for the digital data; in $\S$ III we present the photometric profile of M87; in $\S$ IV we fit theoretical models to the luminosity profile and give the model-independent argument for a central supermassive object; and in $\S \mathrm{V}$ we present a discussion.

\section{OBSERVATIONS AND REDUCTION PROCEDURES}

The two-dimensional SIT detector system has been described in Westphal, Kristian, and Sandage (1975). The raster scan on the silicon target yields $256 \times 256$ 
pixels, each $47 \mu \mathrm{m}$ square. Photometry with the SIT has demonstrated an accuracy of $0.03 \mathrm{mag}$. The CCD was made by Texas Instruments for JPL under NASA contract $7-100$. The array contains $400 \times 400$ pixels, each $23 \mu \mathrm{m}$ square, and is used in a direct mode with no gain. This is made possible by the very low readoutnoise characteristics of the device. Unpublished photometry by Kristian and Westphal over a $12 \mathrm{mag}$ range has shown no departure from linearity, and agreement with a photoelectric sequence by Sandage to within $2 \%$. Laboratory tests of the CCD and internal deviations on the standard stars suggest that the CCD itself has a photometric accuracy of $1 \%$ or better.

The results for M87 presented here are based on three separate observing runs with a total of 20 exposures in three broad-band colors $(B, V$, and $R)$. A journal of observations and additional information pertinent to the telescopes and detectors are given in Table 1. The SIT observations were made with a variety of exposure times including unsaturated frames of the central regions of M87 and frames in which the center was heavily saturated in order that high signal-to-noise ratio be obtained in the regions of the galaxy at the edge of the frame (at $\left.r=80^{\prime \prime}\right)$. The seeing profile and sky brightness were monitored by interlacing the galaxy observations with exposures of similar length on a field $30^{\prime}$ south of M87. The large dynamic range of the CCD enabled one exposure in each color to yield the desired signal-to-noise ratio over the whole frame without saturation of the central regions. The seeing profiles for the CCD frames were found by using faint stars in the same frame as the galaxy; this was permitted again because of the large dynamic range of the CCD. By choosing blue objects, we ensured that none of the "seeing stars" was one of the coterie of globular clusters encircling M87. The CCD observations in seeing with FWHM $=1^{\prime \prime}$ and pixel size 0.25 provided the best-quality data for the nuclear regions of M87.

The CCD photometer system directly images onto the target diode array which has an extremely uniform pixel spacing because of the manufacturing procedure. The only image distortions in this system are due to the
Ross corrector lens at the 200 inch prime focus, which amount to less than $0.2 \%$ across the detector for nearaxis positions. The image scale was determined by directly measuring the diode separation as $22.875 \mu \mathrm{m}$ and using the known image scale of $11^{\prime \prime} .06 \mathrm{~mm}^{-1}$ to give $0.2530 \pm 0$ ".0003 per pixel. As a check we also measured the length of the M87 jet and compared this with the astrometry of de Vaucouleurs, Angione, and Fraser (1968). From this we found 0".254 \pm 0 0.001 per pixel.

The SIT system uses an electrostatically focused intensifier stage which has pincushion distortion. In addition, inequality of the horizontal and vertical readout deflections gives different image scales in the $x$ and $y$ directions. To remove these distortions we compared the positions of 54 stars in a field near the globular cluster M92 as measured on the SIT and on a prime focus direct photograph. This procedure removed the $6 \%$ quadratic pincushion terms and the $9 \%$ difference in the $x$ and $y$ scales of the SIT to an accuracy of $5 \mu \mathrm{m}(=0,05)$. The SIT frames of M92 used for this analysis were coeval with the M87 data taken in 1975.

The sky brightness level was determined and subtracted from all exposures. Typical sky brightness levels were found to be $\mu_{R}=20.53 \pm 0.02 \mathrm{mag}$ $\operatorname{arcsec}^{-2}, \mu_{V}=21.34 \pm 0.01$, and $\mu_{B}=22.28 \pm 0.02$ for the 1975 SIT data. Mean absorption coefficients for Palomar were used to remove atmospheric extinction.

The data were tied to the Johnson-Morgan system by observation of the $B, V, R$ standards of Sandage (1973). Residuals were $\sim 0.04 \mathrm{mag}$ for the SIT and $\sim 0.02$ mag for the CCD.

The SIT photometry was found to be unreliable near heavily saturated regions, probably because of charge migration effects and bending of the electron readout beam. This border effect extended for $\sim 20$ pixels around the very heavily saturated areas on the long exposures, with the intensity levels perturbed below the true values. The existence of this effect was evident on checking against shorter exposures, and the afflicted data were excised from the analysis. A second check was available from comparison with the

TABLE 1

OBSERVATIONS OF M87

\begin{tabular}{|c|c|c|c|}
\hline & $\begin{array}{c}\text { Data Set 1 } \\
\text { (1975 Mar 14) }\end{array}$ & $\begin{array}{c}\text { Data Set } 2 \\
(1977 \text { Feb 25) }\end{array}$ & $\begin{array}{c}\text { Data Set } 3 \\
\text { (1977 May 22) }\end{array}$ \\
\hline 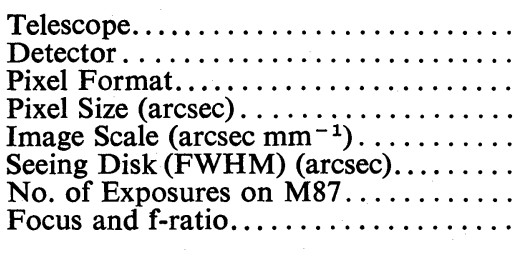 & $\begin{array}{c}200 \text { in. } \\
\text { SIT } \\
256 \times 256 \\
0.52 \\
11.06^{*} \\
1.7 \\
14 \\
\text { Prime focus* } \\
\text { f3.67 }\end{array}$ & $\begin{array}{c}60 \text { in. } \\
\text { SIT } \\
256 \times 256 \\
0.73 \\
15.32 \\
2.0 \\
3 \\
\text { Cassegrain } \\
\text { f8.83 }\end{array}$ & $\begin{array}{c}200 \text { in. } \\
\text { CCD } \\
400 \times 400 \\
0.26 \\
11.06^{*} \\
1.0 \\
3 \\
\text { Prime focus* } \\
\text { f3.67 }\end{array}$ \\
\hline
\end{tabular}

* With Ross corrector lens. 


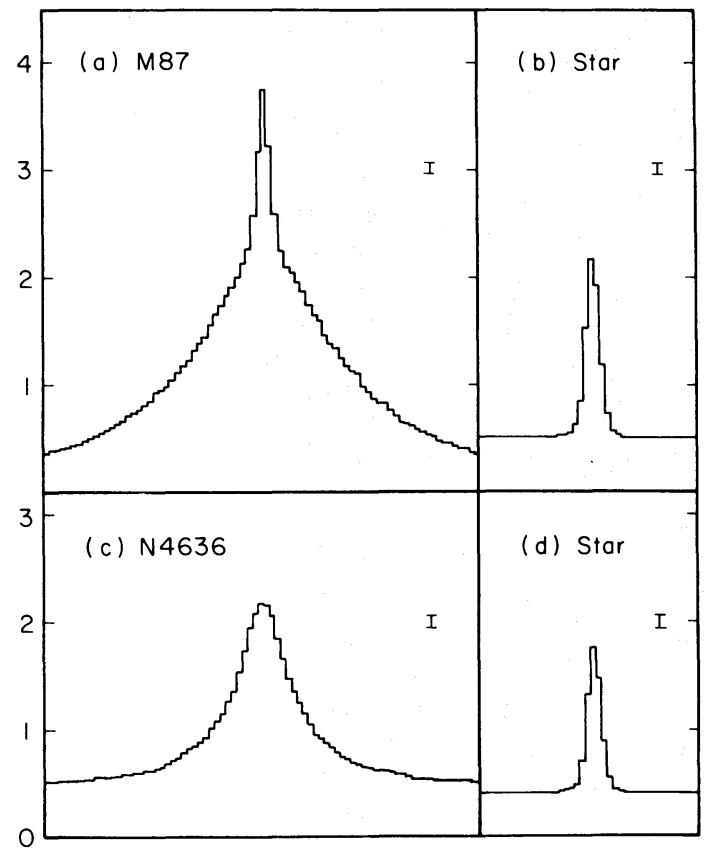

FIG. 1. $-V$ band photometric profiles depicting a $1 \times 80$ pixel slice from the SIT data for (a) M87, showing the abnormal central luminosity spike; (b) a star profile for the M87 data with $\sigma_{*}=0$ "'67; (c) NGC 4636, a typical elliptical galaxy profile; $(d)$ star profile for NGC 4636. Each pixel is $0 " .5$ wide and the intensity scale is linear. Error bars of length $2 \sigma$ are given.

CCD data, which were nowhere saturated; these agreed well with the short-exposure unsaturated SIT frames.

\section{LUMINOSITY PROFILE OF M87}

\section{a) The Luminosity Cusp in M87}

The center of M87 has long been known to contain a small, abnormally bright "nucleus" superposed on the core of the galaxy. This is well shown on the deconvolved IIIa-J plates of Arp and Lorre (1976). Of the other 30 nearby elliptical galaxies for which we have photometric data, none shows a similar feature. To illustrate the bright central cusp in M87, we have plotted a $1 \times 80$ pixel slice across the center in the EW direction from a short exposure SIT frame in the $V$ band. This is shown in Figure 1 together with a similar slice across the galaxy NGC 4636. These galaxies are both classified as type E0 with similar diameters $\left(A_{e}=3\right.$ '. 2 for M87 and $A_{e}=2^{\prime} \cdot 2$ for NGC 4636; de Vaucouleurs, de Vaucouleurs, and Corwin 1976) and are at similar distances [M87 is a principal member of the Virgo $\mathrm{I}(\mathrm{E})$ group at $\Delta=13.6 \mathrm{Mpc}$; NGC 4636 is in the Virgo $X(G 26)$ group at $\Delta=14.3$ $\mathrm{Mpc}$; de Vaucouleurs 1975]. The profile of NGC 4636 was entirely typical of the other 30 galaxies in the survey, with a smooth well-resolved core region. It may be seen in Figure 1, however, that M87 has a bright central luminosity spike scarcely larger than the seeing disk. As we shall see in $\S \mathrm{IV} a$, the anomaly in the luminosity profile of M87 is not confined to the central arcsecond but extends out to $r=20^{\prime \prime}$.

\section{b) Radial Luminosity Profile of M87}

i) The central regions of M87 were inspected on the distortion-free CCD data frames and were found to have no detectable ellipticity $(\epsilon<0.02)$ for $r<30^{\prime \prime}$.

ii) The nuclear luminosity spike was found to be centered to within $0.02(=1.5 \mathrm{pc})$ of the center of M87 as defined by the isophotes for $5^{\prime \prime}<r<30^{\prime \prime}$ (with due allowance for the effects of the optical jet).

Consequently we formed a mean luminosity profile by summing the data in narrow annuli $\left(\sim \frac{1}{2}\right.$ pixel wide) concentric with the central cusp but avoiding the region of the optical jet. A composite profile was then created by using the CCD data for the inner regions, short-exposure SIT data for the intermediate regions, and long-exposure SIT data for the outer regions near the edge of the frame.

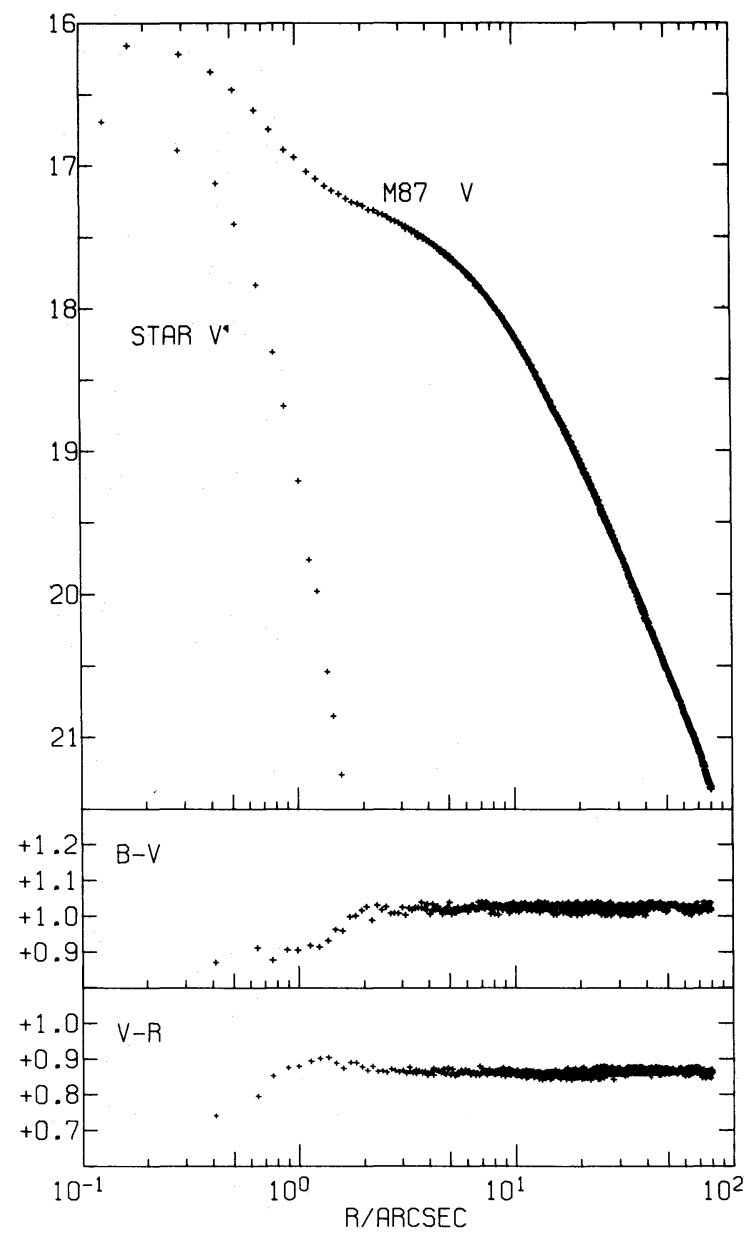

FIG. 2.-SIT/CCD mean luminosity profile of M87 in the $V$ band. The data points have been formed by summing pixels in narrow annuli concentric with the nuclear luminosity spike, and the intensity scale is $\mu_{V}=\operatorname{mag} \operatorname{arcsec}^{-2}$. The instrumental plus seeing profile is represented by the star image extracted from the CCD frame of M87 in the $V$ band. Values of the colors $(B-V)$ and $(V-R)$ inside a simulated $1^{\prime \prime}$ diameter aperture together with the mean annular values outside this aperture are given below the luminosity profile. 
TABLE 2

Photometric DAta For M87

\begin{tabular}{|c|c|c|c|c|c|c|c|}
\hline $\begin{array}{l}r^{\prime \prime} \\
(1)\end{array}$ & $\log _{(2)} r^{\prime \prime}$ & $\begin{array}{l}\mu_{V}^{*} \\
(3)\end{array}$ & $B-V \dagger$ & $V-R \dagger$ & $\begin{array}{l}V_{p} \\
(6)\end{array}$ & $\begin{array}{l}\nu_{V} \\
(7)\end{array}$ & $\begin{array}{l}V_{s} \\
(8)\end{array}$ \\
\hline 0.17 & -0.771 & 16.158 & $\ldots$ & . & $\ldots$ & $\ldots$ & $\cdots$ \\
\hline 0.29 & -0.533 & 16.265 & $\cdots$ & $\cdots$ & $\cdots$ & $\cdots$ & $\ldots$ \\
\hline 0.41 & -0.385 & 16.347 & & & & $\ldots$ & $\cdots$ \\
\hline 0.52 & -0.288 & 16.470 & $0.87 \ddagger$ & $0.74 \ddagger$ & $16.50 \ddagger$ & $\ldots$ & $\ldots$ \\
\hline 0.65 & -0.189 & 16.616 & $0.91^{+}$ & 0.80 & $16.09^{+}$ & $\cdots$ & $\ldots$ \\
\hline 0.76 & -0.119 & 16.750 & 0.88 & 0.85 & 15.80 & $\ldots$ & $\ldots$ \\
\hline 0.89 & -0.052 & 16.895 & 0.91 & 0.88 & 15.55 & $\cdots$ & $\ldots$ \\
\hline 1.00 & -0.002 & 16.946 & 0.90 & 0.88 & 15.36 & $\cdots$ & $\because$ \\
\hline 1.19 & +0.075 & 17.072 & 0.91 & 0.90 & 15.08 & & \\
\hline 1.42 & +0.153 & 17.163 & 0.94 & 0.89 & 14.80 & 19.66 & 15.79 \\
\hline 1.66 & $\begin{array}{r}0.219 \\
\end{array}$ & 17.220 & 0.98 & 0.88 & 14.56 & 19.83 & 15.60 \\
\hline 1.89 & $\begin{array}{r}0.277 \\
\end{array}$ & 17.265 & 1.01 & 0.88 & 14.34 & 19.97 & 15.43 \\
\hline 2.12 & +0.326 & 17.297 & 1.01 & 0.88 & 14.15 & 20.08 & 15.26 \\
\hline 2.36 & $\begin{array}{r}+0.372 \\
\end{array}$ & 17.325 & 1.02 & 0.87 & 13.97 & 20.18 & 15.09 \\
\hline 2.59 & +0.413 & 17.352 & 1.02 & 0.87 & 13.81 & 20.27 & 14.93 \\
\hline 2.83 & +0.452 & 17.385 & 1.01 & 0.86 & 13.65 & 20.35 & 14.77 \\
\hline 3.07 & +0.487 & 17.409 & 1.01 & 0.86 & 13.51 & 20.43 & 14.62 \\
\hline 3.30 & +0.518 & 17.432 & 1.02 & 0.86 & 13.38 & 20.56 & 14.34 \\
\hline 3.70 & +0.568 & 17.477 & 1.03 & 0.86 & 13.18 & 20.61 & 14.26 \\
\hline 4.30 & +0.633 & 17.544 & 1.02 & 0.86 & 12.95 & 20.75 & 13.95 \\
\hline 4.88 & +0.688 & 17.608 & 1.01 & 0.86 & 12.73 & 20.88 & 13.69 \\
\hline 5.47 & +0.738 & 16.672 & 1.02 & 0.86 & 12.52 & 21.01 & 13.44 \\
\hline 6.05 & +0.782 & 17.737 & 1.02 & 0.86 & 12.34 & 21.13 & 13.23 \\
\hline 6.65 & +0.823 & 17.805 & 1.03 & 0.86 & 12.18 & 21.25 & 13.03 \\
\hline 7.23 & +0.859 & 17.875 & 1.03 & 0.86 & 12.05 & 21.36 & 12.86 \\
\hline 7.83 & $\begin{array}{l}+0.894 \\
\end{array}$ & 17.944 & 1.02 & 0.86 & 11.91 & 21.47 & 12.70 \\
\hline 8.41 & +0.925 & 18.010 & 1.02 & 0.86 & 11.79 & 21.58 & 12.55 \\
\hline 8.99 & +0.954 & 18.076 & 1.02 & 0.86 & 11.69 & 21.68 & 12.42 \\
\hline 9.64 & +0.984 & 18.146 & 1.02 & 0.86 & 11.58 & 21.79 & 12.29 \\
\hline 10.6 & +1.024 & 18.249 & 1.02 & 0.86 & 11.45 & 21.95 & 12.11 \\
\hline 11.9 & +1.075 & 18.381 & 1.02 & 0.86 & 11.28 & 22.17 & 11.90 \\
\hline 13.3 & +1.125 & 18.528 & 1.03 & 0.86 & 11.12 & 22.40 & 11.70 \\
\hline 15.0 & +1.176 & 18.678 & 1.02 & 0.86 & 10.96 & 22.65 & 11.51 \\
\hline 16.8 & +1.226 & 18.829 & 1.02 & 0.86 & 10.81 & 22.90 & 11.33 \\
\hline 18.8 & +1.275 & 18.987 & 1.02 & 0.86 & 10.68 & 23.17 & 11.1 \\
\hline 21.1 & +1.325 & 19.149 & 1.02 & 0.86 & 10.55 & 23.45 & 11.00 \\
\hline 23.7 & +1.375 & 19.320 & 1.02 & 0.87 & 10.42 & 23.73 & 10.8 \\
\hline 26.1 & +1.416 & 19.471 & 1.02 & 0.86 & 10.32 & 23.97 & 10.73 \\
\hline 28.3 & +1.452 & 19.584 & 1.02 & 0.86 & 10.24 & 24.19 & 10.6 \\
\hline 31.6 & +1.500 & 19.767 & 1.03 & 0.86 & 10.13 & 24.48 & 10.51 \\
\hline 34.4 & +1.537 & 19.906 & 1.03 & 0.87 & 10.05 & 24.71 & 10.4 \\
\hline 37.1 & +1.569 & 20.017 & 1.03 & 0.87 & 9.99 & 24.89 & 10.3 \\
\hline 39.9 & +1.601 & 20.139 & 1.02 & 0.86 & 9.92 & 25.09 & 10.2 \\
\hline 42.9 & +1.632 & 20.265 & 1.03 & 0.87 & 9.86 & 25.28 & 10.19 \\
\hline 46.3 & +1.666 & 20.401 & 1.03 & 0.86 & 9.79 & 25.48 & 10.12 \\
\hline 50.2 & +1.701 & 20.536 & 1.02 & 0.87 & 9.73 & 25.70 & 10.04 \\
\hline 56.2 & +1.750 & 20.711 & 1.02 & 0.87 & 9.64 & 26.00 & 9.94 \\
\hline 63.1 & +1.800 & 20.903 & 1.02 & 0.86 & 9.54 & 26.30 & 9.84 \\
\hline 71.0 & +1.8 & 21.113 & 1.02 & 0.86 & 9.46 & 26.61 & 9.73 \\
\hline 70 & +1.85 & 21.349 & 1.02 & 0.86 & 9.38 & 26.90 & 9.64 \\
\hline
\end{tabular}

* Formal error is \pm 0.005 ( $1 \sigma$ value).

$\dagger$ Formal error is \pm 0.01 .

$\ddagger$ Values for centered 1 " diameter aperture.

NoTe. $-\mu_{\mathrm{V}}=$ surface brightness in the $V$ band (mag arcsec-2$)$ at radius $r$ from the center of M87; $(B-V),(V-R)=$ surface colors at radius $r ; V_{p}=$ magnitude enclosed by simulated aperture of radius $r ; \nu_{V}=$ spatial luminosity density in mag $\operatorname{arcsec}^{-3} ; V_{s}=$ magnitude enclosed by sphere of radius $r$.

Continuity among the various segments of data was good to 0.01 mag.

The resulting 1995 data points are displayed in Figure 2 and are collected into 144 mean points given in Table 2. Also shown in Figure 2 is a star profile for the CCD data in the $V$ band as derived from a faint star on the $V$ exposure for M87. The Gaussian core,
$I=I_{0} \exp \left(-r^{2} / 2 \sigma_{*}^{2}\right)$, for the star image has a standard deviation $\sigma_{*}=0$ ".46 \pm 0 ".01 or an FWHM $=$ $1^{\prime \prime} .08+0 " .02$

Additional data given in Table 2 are the $V$ magnitudes in simulated circular apertures, the spatial luminosity density, and the magnitude contained within variously sized spheres centered on M87. In 
finding the latter two quantities, we have assumed spherical symmetry for the inversion of the Abel integrals created by fitting analytic functions to the data. We do not give values inside 1".42 where seeing effects start to perturb the profile. All quantities in Table 2 are uncorrected for the seeing profile, since deconvolution procedures merely act as noise magnifiers; seeing effects will be allowed for in subsequent sections by appropriate convolution of theoretical models.

\section{c) Comparison with Previous Photometry}

The CCD/SIT photometry may be compared with the photographic photometry of Oemler (1976) in the $V$ band and Kormendy (1977) in the $G$ band, and with a photoelectric profile in the $B$ band by de Vaucouleurs and Nieto (1977). Color corrections ( $B-$ $V)=+1.02$ and $(G-V)=+0.36$ were used to transform all magnitudes into the $V$ system, and no zero-point corrections were applied. We have plotted the discrepancies $\Delta \mu_{V}=\mu_{V}-\mu_{V}^{*}$, where $\mu_{V}{ }^{*}$ is the SIT/CCD value, in Figure 3 .

The following points may be noted after inspection of Figure 3.

i) The CCD/SIT profile agrees with the photographic photometry of Oemler (1976) for $5^{\prime \prime}<r<$ $80^{\prime \prime}$. The discrepancies are of the order $0.1 \mathrm{mag}$, which is the expected accuracy of the photographic data. A discontinuity of $0.1 \mathrm{mag}$ may be seen in the Oemler data at $r=36^{\prime \prime}$.

ii) The CCD/SIT profile also agrees with the photoelectric data of de Vaucouleurs and Nieto (1977) for $15^{\prime \prime}<r<80^{\prime \prime}$ except for a zero-point discrepancy of $\sim 0.1$ mag.

iii) The data of Kormendy (1977) show systematic discrepancies of up to $\sim 0.4$ mag. While deviations inside $r=1^{\prime \prime}$ may be expected owing to differing

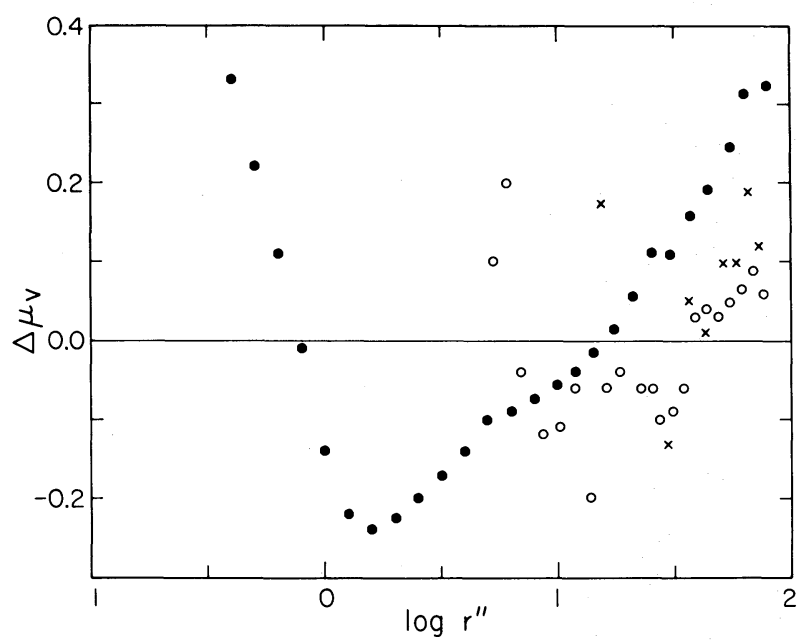

Fig. 3.-Comparison of the SIT/CCD photometry to the photographic of Oemler $(O)$ and Kormendy $(O)$, and the photoelectric data of de Vaucouleurs and Nieto $(x)$. We have plotted the residuals $\Delta \mu_{V}=\mu_{V}-\left.\mu_{V}\right|_{\text {sIT/CCD }}$ with all photometry transformed into the $V$ band and no zero-point corrections applied. seeing profiles and the inability of a photographic plate to cope with strong luminosity gradients, the systematic deviation for $3^{\prime \prime}<r<80^{\prime \prime}$ must cause concern. It cannot be due to an effect of changing color, since it was determined that the $(B-V)$ and $(V-R)$ colors in $\mathrm{M} 87$ are constant in this radial range.

A comparison of the SIT and the CCD profiles showed agreement to within $0.01 \mathrm{mag}$ at all points except inside the central arcsecond, where the higher resolution CCD data rose slightly above the SIT data. In Figure 2 the individual SIT and CCD data points are plotted, and the difference between them is scarcely visible at the $1 \%$ level.

\section{d) Discussion of the SIT/CCD Results}

The following points may be noted after scrutiny of Figure 2.

i) The colors of $\mathrm{M} 87,(B-V)=1.02 \pm 0.01$ and $(V-R)=0.86 \pm 0.01$, are constant for $1^{\prime \prime} .7<r<$ $80^{\prime \prime}$ with $\Delta(B-\bar{V})<0.01$ and $\Delta(V-R)<0.01$.

ii) The bright nucleus is slightly bluer than the rest of the galaxy with $(B-V)=+0.87$ and $(V-R)=$ +0.74 for a simulated 1 " diameter aperture centered on the "cusp."

iii) The star profile has dropped to $1 \%$ of its central intensity at $r=1 " 7$. Because of this, and also because of the constancy of the colors for $r>1^{\prime \prime} .7$, we shall fit theoretical models of the luminosity profile in the range $r>1$ ".7. This will avoid any possible contamination by a possible blue, nonthermal point source in the center. In addition there is no evidence of dust absorption in excess of $A_{v}=0.05 \mathrm{mag}$, and the emission lines of [O II] $\lambda 3737,[\mathrm{~N}$ II] $\lambda 6548,6593$, [S II] $\lambda 6724$ cannot perturb the photometric profile to a significant degree. Since the galaxian colors are constant for $r>1$.'7, we shall assume, for the purpose of model fitting, that the stellar population has a constant mass-luminosity ratio.

\section{COMPARISON WITH THEORETICAL MODELS}

\section{a) Isothermal and King Models}

The modified isothermal sphere models of King (1966) have met with great success in fitting the luminosity profiles of normal elliptical galaxies (King 1975). In the case of M87, however, the deviations from a King model are large and easily observable, since the disagreement extends out to $r=20^{\prime \prime}$. Beyond this radius the King model with a ratio of tidal radius $r_{T}$ to core radius $r_{c} \log r_{T} / r_{c}=2.10$ is a reasonable fit to the luminosity profile of M87. This is shown in Figure 4, where we have fitted such a model to the photographic photometry of Oemler (1976) for $5^{\prime \prime}<r<4$ '.5. Outside $r=4.5$ there is a faint extended corona (de Vaucouleurs and Nieto 1977). The failure of the King model to fit the CCD/SIT photometry in the central regions is shown in Figure 5, where discrepancies of up to $0.25 \mathrm{mag}$ are present for 1 1.7 < $r<10^{\prime \prime}$. It is evident that the observed profile does not curve sharply enough as it rises into the core regions, and then refuses to flatten off as $r \rightarrow 0$, but continues 


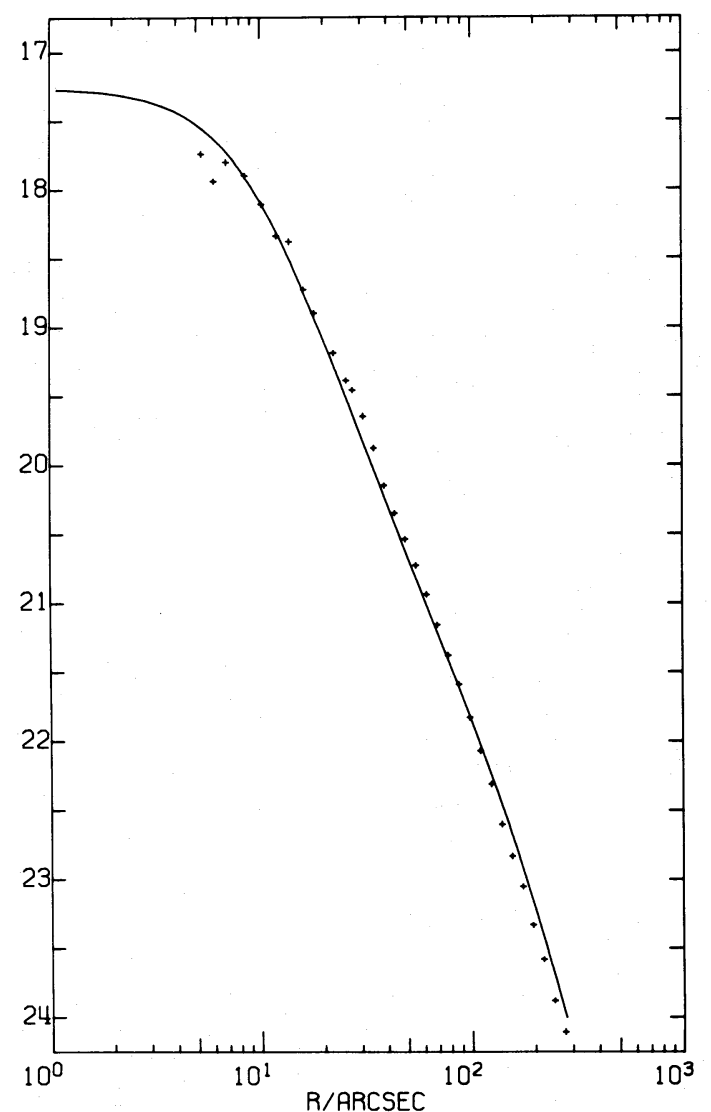

Fig. 4.-The $V$ band photographic data of Oemler (1976) have been fitted with a King model. The core radius is $r_{c}=$ $9 " 6$ and the "tidal" radius is $r_{T}=20 ! 1\left(\log r_{T} / r_{c}=2.10\right)$. The intensity scale is $\mu_{V} \equiv \mathrm{mag} \operatorname{arcsec}^{-2}$.

to rise even at $r=1$."7 where the central luminosity spike begins to be noticeable. The fit cannot be improved by adding a central point luminosity source (see Fig. 5 again), since this cannot affect matters outside $r=1 " .7$.

We may justify the use of single mass King models after the work of Lynden-Bell (1967), who showed that the expected distribution function after galactic collapse has the same velocity dispersion for all stellar masses. Exploratory attempts to fit the luminosity profile of M87 with two mass King models met with limited success. A reasonable fit was obtained by loading the core of the model with a second population of massive stars concentrated inside $r=2^{\prime \prime}$. The total mass required to perturb the profile sufficiently was $\sim 5 \times 10^{9} M_{\odot}$. We shall not pursue this type of model any further because it fails to fit the velocity dispersion data of Sargent et al. (1978); the model predicts a constant velocity dispersion $\sigma_{v}$ for the visible stars across the core of the galaxy, whereas $\sigma_{v}$ is observed to increase sharply as $r \rightarrow 0$.

\section{b) Models with a Large Central Mass}

A stellar dynamics code (VAMPIRE) was written to determine the equilibrium distribution of a stellar

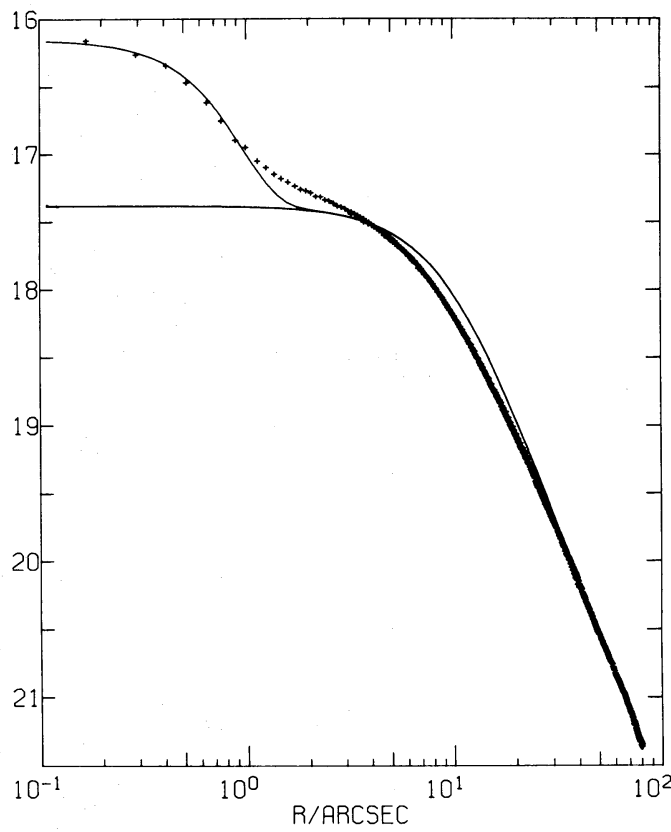

Fig. 5.-An attempt to fit a King model (with $\log r_{T} / r_{c}=$ $2.10)$ to the SIT/CCD $V$ band profile of M87. This is the best fit we could obtain by varying the three parameters of the model. Adding a central point luminosity source improves the fit for $r<1^{\prime \prime}$ but leaves residuals of $0.2 \mathrm{mag}$ for $1^{\prime \prime}<r<20^{\prime \prime}$. The intensity scale is $\mu_{V} \equiv \operatorname{mag} \operatorname{arcsec}^{-2}$.

population around a massive black hole ${ }^{1}$ in a galactic nucleus. This is done by solution of a Fokker-Planck equation in $(E, J)$ space for a multimass stellar population simultaneously with Poisson's equation to determine the relaxed configuration. A minor adaptation allowed the effects of a black hole growing adiabatically in a collisionless stellar population to be studied. These two cases do not differ drastically and result in density profiles $\rho(r) \propto r^{-7 / 4}$ near the black hole for the relaxed models and $\rho(r) \propto r^{-3 / 2}$ for the adiabatic models. We shall not discuss the details of the VAMPIRE models further, since they are described in Young (1977).

A satisfactory fit between the observed profile of M87 and a black hole model is shown in Figure 6. This results in a determination of certain parameters.

i) The core radius of the galaxy,

$$
r_{c}=9.6 \pm 0 " .5=(698 \pm 36) \delta_{15} \mathrm{pc},
$$

where $\delta_{15}=\Delta / 15 \mathrm{Mpc}$ is the distance to M87. Estimates of this distance vary from $\Delta=13.6 \mathrm{Mpc}$ (de Vaucouleurs 1975) to $\Delta=19.5 \mathrm{Mpc}$ (Sandage and Tammann 1974), and we shall include the parameter $\delta_{15}$ where relevant without introducing its uncertainty in the formal error estimates.

${ }^{1}$ A "black hole" in the present context is a nonluminous mass which is pointlike (i.e., which has a size smaller than the seeing disk and the relevant physical length scales). It includes, but does not necessarily imply, the case of a mass which lies within its own Schwarzschild radius. 


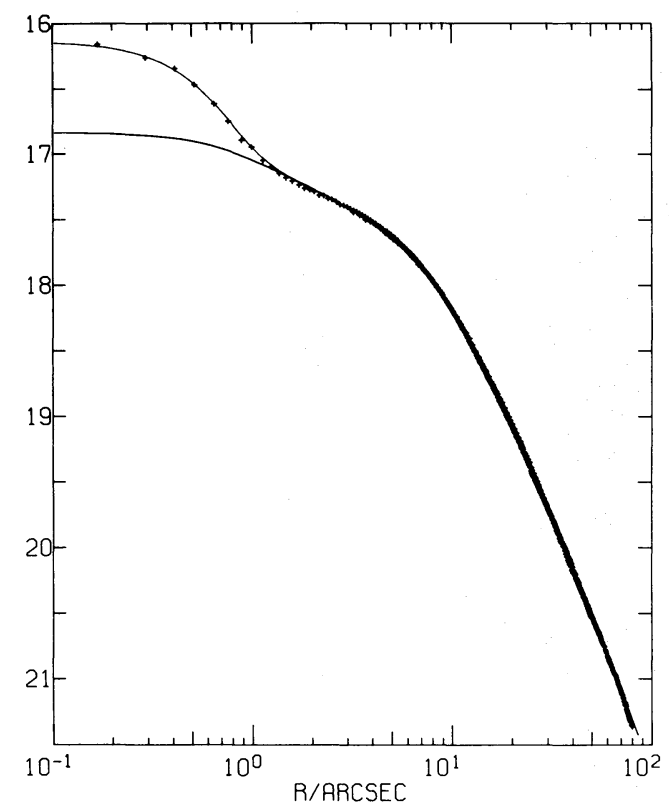

FIG. 6. - The luminosity profile of M87 fitted with a black hole model. An additional point luminosity profile is necessary to fit the data inside $r=1^{\prime \prime} .5$. The deviation of the model from the data is $\leq 0.02 \mathrm{mag}$, giving a black hole mass of $\sim 3 \times 10^{9}$ $M_{\odot}$. The intensity scale is $\mu_{V} \equiv$ mag arcsec ${ }^{-2}$.

ii) The tidal radius is given by

$$
\log r_{T} / r_{c}=2.10 \pm 0.02,
$$

as was determined previously from the data of Oemler (1976). It is by no means clear that the $r_{T}$ represents a true tidal cutoff rather than just a convenient way to truncate the stellar distribution function. However, this parameter has a negligible effect on the profile for $r<10^{\prime \prime}$, wherein lies the action.

iii) Core surface brightness

$$
\mu_{V}=17.20 \pm 0.02 \mathrm{mag} \mathrm{arcsec}^{-2} \text {. }
$$

With an absorption $A_{V}=0.14$ due to our own Galaxy, we deduce the core surface luminosity to be

$$
L_{c}=(5.5 \pm 0.1) \times 10^{3} L_{\odot} \mathrm{pc}^{-2} .
$$

The relation between core surface luminosity $L_{c}$ and core spatial luminosity $L_{0}$ is

$$
L_{c}=2 L_{0} r_{c}
$$

and so we find

$$
L_{0}=(4.0 \pm 0.2) \delta_{15}{ }^{-1} L_{\odot} \mathrm{pc}^{-3}
$$

Since the central luminosity profile is perturbed away from an isothermal sphere by the central black hole, the above parameters are those that would pertain if the black hole were absent.

iv) Mass of black hole

$$
\mu_{\mathrm{H}}=M_{\mathrm{H}} / 4 \pi \rho_{0}\left(r_{c} / 3\right)^{3}=0.6 \pm 0.1
$$

(where $\rho_{0}=$ core mass density). Thus we find

$$
M_{\mathrm{H}}=(4.0 \pm 0.8) \times 10^{8}(M / \mathscr{L}) \delta_{15}{ }^{2} M_{\odot},
$$

where $M / \mathscr{L}$ is the mass-luminosity ratio of the stellar population. The "cusp radius" where the kinetic energy of a typical core star is equal to the potential energy in the gravitational well of the black hole is

$$
r_{a}=\mu_{\mathrm{H}} r_{c}=5 " .8 \pm 1.0=(422 \pm 73) \delta_{15} \mathrm{pc} .
$$

We see that both the core and the central cusp of M87 are well resolved, being 20 and 12 times the seeing disk, respectively. The effects of seeing have been allowed for by appropriate convolution of the model for the fit shown in Figure 6.

v) The observed profile of M87 rises above that predicted by the black hole model inside $r=1.7$. This may be allowed for by adding a point luminosity source to the center with the following magnitude and colors

$$
\begin{aligned}
V_{s} & =16.69 \pm 0.05, \\
(B-V)_{s} & =+0.73 \pm 0.03, \\
(V-R)_{s} & =+0.59 \pm 0.03
\end{aligned}
$$

We assume that the colors of the underlying galaxy remain at their values $(B-V)=+1.02$ and $(V-$ $R)=+0.86$ for $r>1$ ".7. The total luminosity of this point source (in the $V$ band) is

$$
L_{s}=(4.7 \pm 0.2) \times 10^{7} \delta_{15}^{2} L_{\odot} .
$$

vi) The spectroscopic data of Sargent et al. (1978) give a core velocity dispersion of $\sigma_{v}=278 \pm 11 \mathrm{~km}$ $\mathrm{s}^{-1}$. From the relation $\sigma_{v}{ }^{2}=4 \pi G \rho_{0}\left(r_{c} / 3\right)^{2}$, we find the core mass density to be

$$
\rho_{0}=(26 \pm 3) \delta_{15}{ }^{-2} M_{\odot} \mathrm{pc}^{-3},
$$

and the mass-luminosity ratio of the stellar population is

$$
M / \mathscr{L}=(6.5 \pm 0.6) \delta_{15}^{-1} .
$$

The mass of the black hole then becomes

$$
M_{\mathrm{H}}=(2.6 \pm 0.5) \times 10^{9} M_{\odot} .
$$

Our value for $M / \mathscr{L}$ is much lower than previous estimates $(M / \mathscr{L} \sim 50)$, which were based on (1) spuriously high velocity dispersion measurements of $\sigma_{v} \sim 500 \mathrm{~km} \mathrm{~s}^{-1} \quad$ (Minkowski 1962; Brandt and Roosen 1969), and (2) use of the virial theorem applied to the whole galaxy when only the central velocity dispersion was known.

\section{c) Considerations of the Large Nuclear Mass}

A model-independent demonstration of the large nuclear mass in M87 is given in Sargent et al. (1978), along with a general and practical method for determining the mass-luminosity ratio as a function of 
radius given both photometric and velocity dispersion data. Denoting $M(r)$ as the mass enclosed within a sphere of radius $r$, and $\mathscr{L}(r)$ the luminosity similarly enclosed, considerations of the innermost points for which dynamical data exists gave a mass enclosed within 1.5 as

$$
M\left(1^{\prime \prime .5}\right)=(6.5 \pm 1.5) \times 10^{9} \delta_{15} M_{\odot} .
$$

For a $1^{\prime \prime} .5$ radius aperture centered on M87, the integrated magnitude is $V_{p}\left(1^{\prime \prime} .5\right)=14.71 \pm 0.03$. Allowing for projection effects through the galaxy, the magnitude of a $1^{\prime \prime} .5$ sphere is $V_{s}\left(1^{\prime \prime} .5\right)=15.73 \pm$ 0.07 (see Table 2). Allowing for galactic absorption $A_{V}=0.14$, the total luminosity is

$$
\mathscr{L}\left(1^{\prime \prime} .5\right)=(11.2 \pm 0.8) \times 10^{7} \delta_{15}^{2} L_{\odot},
$$

and the mean mass-luminosity ratio enclosed with radius $r$, taking all material into account, is

$$
\langle M / \mathscr{L}\rangle(1.5)=(58 \pm 16) \delta_{15}{ }^{-1} .
$$

This is a factor of 10 higher than for the stellar population in the rest of the core and demonstrates the existence of a large, invisible mass in the nucleus of M87.

According to the black hole model shown in Figure 6 , the mass of stars enclosed within a $1^{11.5}$ sphere is

$$
M_{*}(1 " .5)=(4.2 \pm 0.3) \times 10^{8} \delta_{15} M_{\odot},
$$

and their luminosity is

$$
\mathscr{L}_{*}\left(1{ }^{\prime \prime} .5\right)=(6.5 \pm 0.3) \times 10^{7} \delta_{15}{ }^{2} L_{\odot} .
$$

If the residual "anomalous" mass and luminosity of

$$
\begin{aligned}
& M_{s}(1.5)=(6.1 \pm 1.5) \times 10^{9} \delta_{15} M_{\odot} \\
& \mathscr{L}_{s}(1.5)=(4.7 \pm 0.2) \times 10^{7} \delta_{15}{ }^{2} L_{\odot}
\end{aligned}
$$

are associated, then the mystery object has a massluminosity ratio of

$$
(M / \mathscr{L})_{s}=(130 \pm 38) \delta_{15}^{-1}
$$

in solar units employing the $V$ band.

\section{d) Nature of the Central Luminosity Source}

The present data do not define the nature of the central luminous source required to fit the black hole model of $\S I V b$ above, although there are additional pertinent observations.

i) Spectroscopic data (Sargent et al. 1978) show the central regions of M87 to have a late-type stellar spectrum. The change in line strength observed across the nucleus is a barely significant $4 \%$. This places a limit of $V=16.4$ on the luminosity of a nonthermal continuum source in the center of M87.

ii) The nucleus of M87 is unpolarized, whereas the "jet" is linearly polarized at a level of $20 \%$ (Angel 1977, private communication).
On morphological grounds, one might expect blue nonthermal emission akin to that observed in a Seyfert galaxy, and we note that the luminosity of the central point source is $V=16.7$, which does not violate the constraint given above. However, the colors and slight decrease in line strength would also be consistent with a population of $\mathrm{G} 5$ stars.

\section{SUMMARY AND DISCUSSION}

We have demonstrated the following points.

i) The core radius of M87 is $r_{c}=9.6=700 \mathrm{pc}$ and the core surface brightness is $\mu_{V}=17.20 \mathrm{mag}$ $\operatorname{arcsec}^{-2}=5.5 \times 10^{3} L_{\odot} \mathrm{pc}^{-2}$. The core luminosity is then $L_{0}=4.0 L_{\odot} \mathrm{pc}^{-3}$.

ii) The colors of M87 are constant at $(B-V)=$ +1.02 and $(V-R)=+0.86$ for $1^{\prime \prime} 7<r<80^{\prime \prime}$. The central regions are slightly bluer than the rest of the galaxy with $(B-V)=+0.87$ and $(V-R)=+0.74$ for a centered $1^{\prime \prime}$ diameter aperture.

iii) The luminosity profile cannot be fitted by isothermal or King models (with constant mass-luminosity ratio) because the central regions $\left(r<10^{\prime \prime}\right)$ contain a bright luminosity cusp. This cusp is not seen in other nearby, normal elliptical galaxies and so adds to the peculiarities exhibited by M87.

iv) In the radial range $1^{\prime \prime} 7<r<80^{\prime \prime}$ the luminosity profile of M87 can be fitted by a King model containing a central black hole of mass $M_{\mathrm{H}}=3 \times 10^{9} M_{\odot}$. An additional point luminosity source with $V=16.69$, $(B-V)=+0.73$ and $(V-R)=+0.59$ is successful in fitting the profile and colors for $r<1$ ".7.

v) Using the core velocity dispersion $\sigma_{v}=278$ $\mathrm{km} \mathrm{s}^{-1}$ found by Sargent et al. (1978), we find for M87 a core mass density of $\rho_{0}=26 M_{\odot} \mathrm{pc}^{-3}$ and a mass-luminosity ratio $M / \mathscr{L}=6.5$ (in solar units) for the stellar population. The velocity dispersion increases to $350 \mathrm{~km} \mathrm{~s}^{-1}$ as $r \rightarrow 0$ in accord with the black hole model.

vi) $\mathrm{A}$ model-independent dynamical analysis of the central regions finds the total mass enclosed by a 1 1.5 sphere centered in M87 to be $M\left(1^{\prime \prime} .5\right)=6.5 \times$ $10^{9} M_{\odot}$. Since the luminosity is $L\left(1^{\prime \prime} .5\right)=11.2 \times$ $10^{7} L_{\odot}$, the mean mass-luminosity ratio is $M / \mathscr{L}\left(1^{\prime \prime} .5\right)=$ 58 , a factor of 10 higher than for the stellar population for $r>1^{\prime \prime} 7$.

In summary, the data show that the nucleus of M87 contains a supermassive object of mass $M_{\mathrm{H}} \sim 5 \times$ $10^{9} M_{\odot}$, radius $r \leq 100 \mathrm{pc}$, and $M / \mathscr{L} \geq 60$. Various suggestions may be made as to the possible nature of this object.

1. A small, dense star cluster with a high $M / \mathscr{L}$ may be bound in the core of M87. Suitable objects in the cluster include white dwarfs, neutron stars, black holes, or perhaps even extreme $\mathbf{M}$ dwarfs. Such a cluster, as a dynamically bound entity, would have to have a very high velocity dispersion $\left(\sigma_{v}>1000 \mathrm{~km}\right.$ $\mathrm{s}^{-1}$ ) and could therefore mimic a continuum source by virtue of broadening the stellar spectral lines to invisibility (although one could still detect the metallicity break at $3900 \AA$ ). This central cluster cannot be akin to the small nucleus seen in M31 (Kinman 1965), 
since the M87 "cluster" would contain 100 times as much mass and is associated with changes in $M / \mathscr{L}$ and increases in the stellar velocity dispersion (which the M31 cluster is not; Morton, Andereck, and Bernard 1977).

It is unlikely that such a cluster would have been formed by the spiraling in of companion galaxies or globular clusters as suggested for M31 by Tremaine, Ostriker, and Spitzer (1975). The objections are: (i) galaxies could not form an object less than or only $100 \mathrm{pc}$ across; (ii) the total mass in the globular clusters of M87 is $\sim 10^{9} M_{\odot}$; thus 5 times this amount would already have had to spiral in; and (iii) neither galaxy or globular cluster material could yield $M / \mathscr{L} \geq 60$.

2 . Since the relaxation time scale in the core of M87 is $t_{R} \sim 10^{14} \mathrm{yr}$, it is unlikely that the central cusp is incipient gravothermal collapse (Lynden-Bell and Wood 1968) or runaway mass segregation (Spitzer 1969). In any case, since the stellar population remains close to dynamical equilibrium during such events, the arguments of $\S$ IV $c$ still apply.

3. It may be possible to explain the photometric and dynamical data if the stellar population is out of dynamical equilibrium. To throw the stars out of equilibrium, however, one must contrive to remove or add a mass $\sim 5 \times 10^{9} M_{\odot}$ in a dynamical time scale of $\sim 10^{5} \mathrm{yr}$; furthermore, the disturbance will die away in this dynamical time scale of $10^{5} \mathrm{yr}$. It is possible to imagine a slingshot mechanism (Saslaw, Valtonen, and Aarseth 1974) removing $\sim 5 \times 10^{9} M_{\odot}$ from the nucleus of M87, with the resulting ejecta forming the optical jets.

4. Another possibility is that the nucleus of M87 contains a single compact object such as a spinar or massive black hole. It should be emphasized that we are not forced to such a model on either observational or theoretical grounds, but it is consistent with all the data, and is in many ways the most attractive of the models considered. A central black hole could easily fuel itself by consuming mass lost from stars in the cusp to explain the $\sim 10^{42}$ ergs $\mathrm{s}^{-1}$ energy output of Virgo A. A mass flux of $\sim 10^{-2} M_{\odot} \mathrm{yr}^{-1}$ is possible and so an efficiency of conversion to radiation of only 0.002 would be necessary.

M87 is at present probably the most plausible case for a massive black hole in a galaxy nucleus. The existing data are pressing upon the current limits for ground-based observations, and are not likely to be improved upon in the near future. Probably the best hope for a dramatic improvement in the data lies with the Space Telescope, which will offer an order of magnitude increase in resolution for more detailed studies of M87 and a search for similar phenomena in more distant supergiant elliptical radio galaxies.

We thank John Bahcall, Roger Blandford, Peter Goldreich, Jim Gunn, Vincent Icke, John Kormendy, Russell Redman, Martin Rees, Scott Tremaine, and Gerard de Vaucouleurs for discussions and valuable assistance. We also thank Wallace Sargent, Alec Boksenberg, Keith Shortridge, Roger Lynds, and Fred Hartwick for the close cooperation involved in analyzing the M87 data presented in this and the accompanying paper. This work was supported in part by the National Science Foundation, under grant MPS 75-16327 to the Carnegie Institution of Washington.

\section{REFERENCES}

Arp, H. C., and Lorre, J. 1976, Ap. J., 210, 58.

Brandt, J. C., and Roosen, R. G. 1969, Ap. J. (Letters), 156, L59.

Cohen, M. H., Moffet, A. T., Shaffer, D., Clark, B. G., Kellermann, K. I., Jauncey, D. L., and Gulkis, S. 1969, Ap. J. (Letters), 158, L83.

de Vaucouleurs, G. 1975, in Galaxies and the Universe, ed. A. Sandage, M. Sandage, and J. Kristian (Chicago: University of Chicago Press), p. 557.

de Vaucouleurs, G., Angione, R., and Fraser, C. W. 1968, Ap. Letters, 2, 141.

de Vaucouleurs, G., de Vaucouleurs, A., and Corwin, H. G., Jr. 1976, Second Reference Catalogue of Bright Galaxies (Austin: University of Texas Press).

de Vaucouleurs, G., and Nieto, J. L. 1977, preprint.

Felten, J. E. 1968, $A p . J ., 151,861$.

Felten, J. E., Arp, H. C., and Lynds, C. R. 1970, Ap. J., $159,415$.

Kellermann, K. I., Clark, B. G., Cohen, M. H., Shaffer, D. B., Broderick, J. J., and Jauncey, D. L. 1973, Ap. J. (Letters), 179, L141.

King, I. R. 1966, A.J., 71, 64.

1975, in Dynamics of Stellar Systems, IAU Symposium No. 69, ed. A. Hayli (Dordrecht: Reidel), p. 99.

Kinman, T. D. 1965, Ap. J., 142, 1376.

Kormendy, J. 1977, Ap. J., 214, 359.

Lynden-Bell, D. 1967, M.N.R.A.S., 136, 101

Lynden-Bell, D. 1969, Nature, 223, 690

Lynden-Bell, D., and Wood, R. 1968, M.N.R.A.S., 138, 495

Minkowski, R. 1962, in Problems of Extragalactic Research, IAU Symposium No. 15, ed. G. C. McVittie (New York: Macmillan), p. 112.

Moffet, A. T. 1975, in Galaxies and the Universe, ed. A Sandage, M. Sandage, and J. Kristian (Chicago: University of Chicago Press), p. 211.

Morton, D. C., Andereck, C. D., and Bernard, D. A. 1977, Ap. J., 212, 13.

Oemler, A., Jr. 1976, Ap. J., 209, 693.

Sandage, A. 1973, Ap. J., 183, 711.

Sandage, A., and Tammann, G. A. 1974, Ap. J., 194, 559.

Sargent, W. L. W., Young, P. J, Boksenberg, A., Shortridge K., Lynds, C. R., and Hartwick, F. D. A. 1978, Ap. J., 221, in press.

Saslaw, W. C., Valtonen, M. J., and Aarseth, S. J. 1974, Ap. J., 190, 253.

Spitzer, L. 1969, Ap. J. (Letters), 158, L139.

Tremaine, S. D., Ostriker, J. P., and Spitzer, L., Jr. 1975 , Ap. J., 196, 407.

Westphal, J. A., Kristian, J., and Sandage, A. 1975, Ap. J. (Letters), 197, L95.

Young, P. J. 1977, "Massive Black Holes in Galactic Nuclei," presented at NATO Conference "Energy Sources and Emission Mechanisms in QSO's," Cambridge.

Note added in proof.-G. de Vaucouleurs has kindly sent us his new photographic results, obtained with J. L. Nieto, for the $\mu_{B}$ profile of M87 at small radii. These were compared with $\mu_{B}=\mu_{V}+(B-V)$ measured by the SIT/CCD. After subtracting a zero-point difference of $0.1 \mathrm{mag}$, the difference in the profiles is less than $0.03 \mathrm{mag}$ 
for $1^{\prime \prime}<r<80^{\prime \prime}$. For $r<1^{\prime \prime}$, deviations of $0.1 \mathrm{mag}$ occur due to the higher resolution of the SIT/CCD data (seeing $\sigma_{*}=0 "$.46 against $\sigma_{*}=0.7$ for the McDonald photographic data). In particular, the McDonald data also demonstrate the shape of the M87 profile as shown in Figure 2, with the steepness of the slope even in the core 1 regions of the galaxy and the reversal in sign of the second derivative of $\mu(\log r)$ between $r=1^{\prime \prime}$ and $r=2 \frac{1}{2}$.

J. A. KRISTIAN: Hale Observatories, 813 Santa Barbara Street, Pasadena, CA 91101

F. P. LANDAUER: Jet Propulsion Laboratory, 4800 Oak Grove Drive, Pasadena, CA 91103

J. A. WestPhal: Division of Geological and Planetary Sciences, California Institute of Technology, Pasadena, CA 91125

C. P. WILSON: 5235 James Road, Santa Barbara, CA 93111

P. J Young: Robinson Laboratory of Astrophysics, California Institute of Technology, Pasadena, CA 91125 